This document is the unedited Author's version of a Submitted Work that was subsequently accepted for publication in ACS Sensors, copyright (c) American Chemical Society after peer review. To access the final edited and published work see: https://dx. doi.org/10.1021/acssensors.8b00393. 


\title{
Label-free and real-time detection of tuberculosis in human urine samples using a nanophotonic point-of-care platform
}

\author{
Patricia Ramirez-Priego ${ }^{\dagger}$, Daan Martens ${ }^{\star, \S}$, Ayssar A. Elamin", Pieterjan Soetaert ${ }^{\perp}$, Wim Van Roy ${ }^{\#}$, Rita \\ Vos $^{\#}$, Birgit Anton ${ }^{\nabla}$, Ronny Bockstaele ${ }^{\perp}$, Holger Becker ${ }^{\nabla}$, Mahavir Singh", Peter Bienstman ${ }^{\ddagger}$, and \\ Laura M. Lechuga*,† \\ †Nanobiosensors and Bioanalytical Applications Group, Catalan Institute of Nanoscience and Nanotechnology (ICN2), \\ CSIC, BIST and CIBER-BBN, Campus UAB, Bellaterra, 08193 Barcelona, Spain \\ †Photonics Research Group, Ghent University/imec, Technologiepark-Zwijnaarde 15, 9052 Ghent, Belgium \\ ${ }^{\S}$ Center for Nano- and Biophotonics, Ghent University, Technologiepark-Zwijnaarde 15, 9052 Ghent, Belgium \\ "LIONEX Diagnostics and Therapeutics GmbH, Salzdahlumer Str. 196, Building 1A, 38126 Braunschweig, Germany \\ ${ }^{\perp}$ Trinean NV, Dulle-Grietlaan 17/3, 9050 Ghent, Belgium \\ \#imec, Kapeldreef 75, 3001 Leuven, Belgium \\ ${ }^{\nabla}$ microfluidic ChipShop GmbH, Stockholmer Str. 20, 07747 Jena, Germany
}

\section{KEYWORDS: point-of-care; nanophotonic biosensor; LAM; biomarker; urine; diagnosis; tuberculosis}

\begin{abstract}
Tuberculosis (TB) is the leading global cause of death from a single infectious agent. Registered incidence rates are low, especially in low-resource countries with weak health systems, due to the disadvantages of current diagnostic techniques. A major effort is directed to develop a point-of-care (POC) platform to reduce TB deaths with a prompt and reliable low-cost technique. In the frame of the European POCKET Project, a novel POC platform for the direct and non-invasive detection of TB in human urine was developed. The photonic sensor is integrated in a disposable cartridge and is based on a highly sensitive Mach-Zehnder Interferometer (MZI) transducer combined with an on-chip spectral filter. The required elements for the read-out are integrated in an instrument prototype, which allows real-time monitoring and data processing. In this work, the novel POC platform has been employed for the direct detection of lipoarabinomannan (LAM), a lipopolysaccharide found in the mycobacterium cell wall. After the optimization of several parameters, a limit of detection of $475 \mathrm{pg} / \mathrm{mL}(27.14 \mathrm{pM})$ was achieved using a direct immunoassay in undiluted human urine in less than 15 minutes. A final validation of the technique was performed using twenty clinical samples from TB patients and healthy donors, allowing the detection of TB in people regardless of HIV coinfection. The results show excellent correlation to those obtained with standard techniques. These promising results demonstrate the high sensitivity, specificity and applicability of our novel POC platform, which could be used during routine check-ups in developing countries.
\end{abstract}

Tuberculosis (TB) is a re-emerging infectious disease caused by Mycobacterium tuberculosis affecting millions of people worldwide. According to the 2017 World Health Organisation (WHO) report, ${ }^{1}$ TB infected 10.4 million people, resulting in 1.7 million deaths with more than $95 \%$ of these cases occurring in developing countries. TB is considered the leading fatal infectious agent, surpassing human immunodeficiency virus (HIV) and acquired immune deficiency syndrome (AIDS). ${ }^{1}$ Two types of TB have been identified: active and latent. In both cases, the bacterium is present in the human body. In active TB, the bacterium is constantly multiplying, invading diverse organs with a high infective potential. On the other hand, latent $\mathrm{TB}$ is asymptomatic and the bacterium is inactive without the ability to infect others.
Traditional methods for the detection of active TB include culture testing as the current reference standard and sputum smear microscopy. However, both of them are imprecise, slow and require qualified personnel and laboratory equipment. ${ }^{1-3}$ Since 2010, WHO began to recommend the use of a new molecular assay, GeneXpert (Cepheid, US), based on nucleic acid amplification for the detection of M. tuberculosis and for the determination of its resistance to rifampicin, the common antibiotic employed to treat several infectious diseases, including TB. ${ }^{1,4}$ Although promising at the beginning, GeneXpert has not improved the TB screening capabilities as compare to conventional tests; also it incurs infrastructure limitations in developing countries, as it needs a controlled temperature environment, with the addition of the instrumentation, reagents and cartridges costs. $^{1,5}$ Therefore, there is still an urgent need to develop a 
highly sensitive and specific point-of-care (POC) platform for the rapid diagnosis of TB in low-resource countries; a platform that should be cost efficient, user-friendly, non-invasive and requiring minimal biosafety standards. ${ }^{6-8}$

Several $M$. tuberculosis secreted antigens have been described as biomarkers in TB, such as lipoarabinomannan (LAM), antigen 85 complex (Ag85), ESAT-6, CFP-10 and MPT64. ${ }^{7,9-11}$ These antigens can be used for the direct detection of active TB in different biological samples other than sputum, such as urine and plasma. Of these, urine is potentially the best candidate for a POC analysis as it can be readily obtained from children and adults, can be easily stored, has a low infection risk during sample collection and does not require a laboratory with a high biosafety level. ${ }^{7,12,13}$

LAM is a widely known and structurally important $17.5 \mathrm{kDa}$ lipopolysaccharide found in mycobacterial cell walls. ${ }^{12,14-16}$ When $M$. tuberculosis is lysed by the host immune system, it is filtered by the kidneys and LAM can then be detected in the urine. ${ }^{15}$ Since no human glycosidases are known for the degradation of LAM, an antigen with glycosidic linkages, it is a key potential biomarker in urine. ${ }^{13}$ Several studies have confirmed the presence of LAM antigens in the urine of patients with active $\mathrm{TB}^{12,17}$ and have found that the amount of LAM in urine is correlated with the bacterial burden metabolic activity and rate of degradation of the bacteria, and hence allows for a semiquantitative assessment of the infection. Also have found that LAM antigens play a key role in phagocyte evasion, ${ }^{17}$ modulation of the host response during infection, ${ }^{18}$ inhibition of mycobacterial antigen processing, ${ }^{17}$ production of tumour necrosis factor $^{17}$ and growth and pathogenesis of M. tuberculosis ${ }^{19}$. Detection of LAM in urine has several advantages in comparison to the conventional techniques. There are ELISA and lateral flow tests commercially available for the detection of LAM in urine ${ }^{14,20}$ with adequate diagnostic specificity of $98 \%$ to $99 \%,{ }^{21}$ but unsatisfactory low sensitivity for TB patients who do not have HIV. These techniques achieve higher sensitivity (61 $67 \%$ ) for people with less than 50 cluster of differentiation four (CD4) cells/ $\mu \mathrm{L}$, who are seriously ill HIV-positive patients, than for patients only infected with TB $(4-8 \%) .{ }^{14,21}$ Taking into account that TB is the leading cause of death of HIV-positive people with more than $40 \%$ of incidents worldwide ${ }^{1}$ and that the current methods are not able to detect TB in people without $\mathrm{HIV},{ }^{20}$ effort is necessary to develop new methodologies for TB diagnosis in patients with and without HIV in order to reduce the number of TB cases and deaths, especially in developing countries.

We present the optimisation and validation of a novel POC platform for the direct and non-invasive detection of TB in human urine. This new POC platform was developed in the frame of a European project (www.pocket-proj.eu) and the complete description of the new instrument can be found $\mathrm{in}^{22}$. The photonic sensor chip is based on a highly sensitive Mach-Zehnder Interferometer (MZI) transducer combined with an on-chip spectral filter (see Figure 1). ${ }^{23}$ The sensor chip is integrated in a disposable polymer microfluidic cartridge. A superluminiscent diode (SLED) is used as the light source whereas a CMOS sensor is employed for the signal read-out, both of which are integrated in the instrument prototype. This instrument also incorporates a pumping unit and a graphical user interface that allows real-time monitoring, data processing and controlling of fluid injection into the sensor cartridge. Prior to use, the sensor surface is functionalised with high-quality and selective monoclonal antibodies against LAM. Using this platform, TB detection is achieved in undiluted human urine samples in less than 15 minutes by employing a direct immunoassay. This demonstrates the high sensitivity, specificity and applicability of our platform for TB diagnosis surpassing the existing limitations of the current systems.

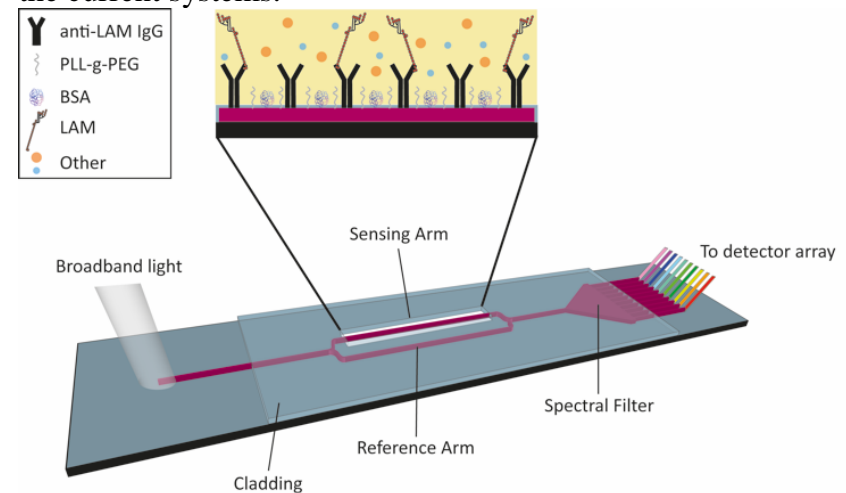

Figure 1. Layout of the photonic sensor chip based on MachZehnder Interferometer transducers and read-out by an on-chip spectral filter. Zoom in the sensing area shows a scheme of the biosensing interaction of the anti-LAM/LAM immunoreagents.

\section{MATERIALS AND METHODS}

Biological samples. Stored urine samples from Tanzania used for the validation of TB diagnosis technique. Twenty urine samples were used for this study, including 10 healthy donors, five TB patients with HIV and five TB patients without HIV. All samples were previously analysed using GeneXpert (Cepheid, US) and sputum smear microscopy.

Prototype instrument. The photonic chip $(7.16 \times 6.76 \mathrm{~mm})$ is fabricated in silicon nitride at wafer-scale in a clean room facility, integrates six MZI sensors and a reference sensor (see Figure 2A). The reference sensor is used to compensate the dispersion of the grating couplers, the light source and the on-chip spectral filter, resulting in an accuracy increase of the response.

A complete description of the new POC instrument can be found $\mathrm{in}^{22}$. Briefly, a SLED $(850 \mathrm{~nm})$ broadband light source is coupled to each sensor simultaneously using a separated grating coupler. The output of each MZI sensor is connected to an arrayed waveguide grating (AWG) with 30 channels which work as on-chip spectral filter. Another separated grating couplers are used to couple the light from the 30 output channels of the AWG to a CMOS camera which monitors the intensity of the different spectral channels simultaneously.

The photonic chip was placed in a disposable cartridge (75.5 $\mathrm{x} 25.5 \mathrm{~mm}$ ) which is fabricated with cyclic olefin copolymer (COC) as shown in Figure 2B. The input and output grating couplers are left exposed to avoid variations in the optical pathways. A microfluidic channel delivers the sample onto the MZI sensing arms. A waste reservoir able to handle a maximum volume of $1 \mathrm{~mL}$ is connected at the end of the fluidic path. The cartridge includes three female Luer interfaces to facilitate connections with standard syringes employed for the injection of urine samples and the required buffers. The fluidic system includes a special bubble trap to avoid undesired air bubbles, and an optional urine filter ( $5 \mu \mathrm{m}$ pore size). 

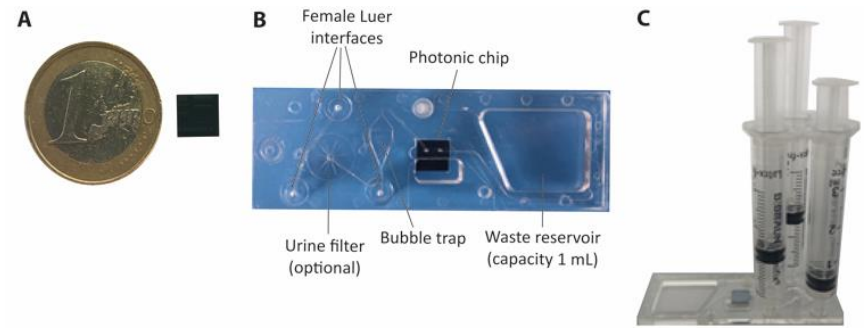

Figure 2. (A) Photonic sensor chip. (B) Microfluidic cartridge integrated with the photonic sensor chip. (C) Microfluidic cartridge employing three-syringe system.

The complete instrument $(22 \times 22 \times 49.30 \mathrm{~cm})$ contains the optical system, the pumping units and a touch screen as a user interface, as shown in Figure S-1. The sensors are evaluated by measuring wavelength shifts due to surface refractive index changes.

The sensorgram of each sensor is graphically displayed in real-time providing the user with immediate feedback of the patient sample diagnosis. Additionally, the interface enables the control of the pump speed and time of injection for each syringe and bubble trap monitoring.

Due to the volume limitations of the cartridge waste reservoir and the three-syringe configuration in the final POC configuration (Figure 2C), we decided to modify the instrument with an in-flow system in order to perform its complete analytical characterisation at the laboratory level, prior to the evaluation of the final POC instrument. To include this in-flow system we have added: (i) a syringe pump (NewEra, US) with adjustable pumping speed, (ii) a two-position valve (VICI, US) that allows sequential loading of the sample loop and injection into the cartridge, and (iii) three Teflon caps to provide connections to the cartridge female Luer interfaces. One of these caps allows the flow of the solution in the cartridge and the other two form a vacuum to avoid air bubbles. The evaluation of real samples with the new POC platform was performed using both, the inflow and the three-syringe configuration.

Antibody immobilization and direct immunoassay. For the detection of LAM, a monoclonal IgG antibody against LAM (anti-LAM) was developed within the framework of the project by LIONEX GmbH (Germany). The antibody was diluted in PBS and immobilised by physical adsorption onto the surface of the photonic sensor chip, followed by a blocking step to avoid non-specific adsorptions. Milli-Q water was used as the running buffer at a flow rate of $10 \mu \mathrm{L} / \mathrm{min}$.

Once the immobilisation process was completed, an immunoassay was performed which allows the direct detection of LAM from the sample. The running buffer was changed to PBS. In order to obtain a complete calibration curve, different LAM concentrations $(1,10,50,100,250,500,750$ and $1000 \mathrm{ng} / \mathrm{mL})$ were employed. An additive assay was performed by flowing successive dilutions of increasing LAM concentrations.

\section{RESULTS AND DISCUSSION}

Evaluation of the bulk sensitivity. The estimation of the bulk sensitivity of the photonic sensor chip to changes in the refractive indices of a solution over its sensing area provides a preliminary evaluation of the performance and reproducibility of our device and the prototype instrument.

In order to analyse the performance of the sensor chip, different solutions of PBS $(0.5 x, 1 x, 2.5 x, 5 x, 7.5 x$ and $10 x)$, which do not chemically modify the sensor surface, were sequentially injected providing minute changes in their refractive indices in contrast with the milli-Q water employed as running buffer $\left(\Delta \eta_{\text {PBS 0.5x }}=1 \cdot 10^{-3}\right.$ RIU, $\Delta \eta_{\text {PBS } 1 \mathrm{x}}=1.70 \cdot 10^{-3}$ RIU, $\Delta \eta_{\text {PBS } 2.5 \mathrm{x}}=$ $4.50 \cdot 10^{-3}$ RIU, $\Delta \eta_{\text {PBS } 5 x}=8.20 \cdot 10^{-3}$ RIU, $\Delta \eta_{\text {PBS } 7.5 x}=1.21 \cdot 10^{-2}$ RIU, $\Delta \eta_{\text {PBS 10x }}=1.56 \cdot 10^{-2}$ RIU) (see Figure 3 ). The refractive indices from the different PBS concentrations were determined with a digital refractometer (Rudolph Research Analytical, US). The noise of the system was very low $(0.003 \mathrm{~nm})$ and was determined as the standard deviation of a blank signal in which only running buffer was flowing.

A linear dependence is observed in Figure 3 between the refractive index change and the measured wavelength shift variation of the sensor output. The calibration for the bulk refractive index changes was fitted to a linear model $(\mathrm{R} 2=0.99)$, resulting in a limit of detection (LOD) as low as $3 \cdot 10^{-6}$ RIU. This evaluation was repeated using different sensor cartridges, and resulted in the same LOD, demonstrating in this way the excellent performance and reproducibility of the sensor chips and the POC platform.

Production of anti-LAM and LAM. The proprietary mouse anti-LAM IgG is specific monoclonal antibodies to mycobacterial LAM with high affinity towards $M$. tuberculosis LAM.

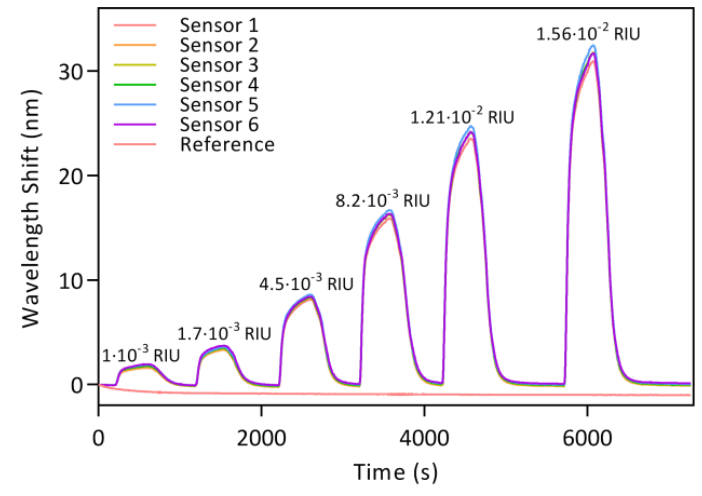

Figure 3. Calibration curve of the sensor chips by flowing solutions of PBS with different refractive indices. The top of each bulk change specifies the $\Delta \eta$ that is evaluated $\left(\eta_{[\mathrm{PBS}]}-\eta_{\mathrm{H} 2 \mathrm{O}}\right)$.

The antibody was purified from defined hybridoma cells culture by affinity chromatography. The quantity of antibody was determined using the Lowry protein assay and the purity of the antibody was analysed using SDS gel electrophoresis, western blotting, Tapestation and exclusion chromatography. The antibody preparation purity was determined to be $95 \%$ pure. Specific antigen-antibody binding was determined by western blot utilising purified M. tuberculosis LAM (LIONEX, Germany) (Figure S-2) and ELISA.

Furthermore Bio-Layer Interferometry (BLI) was used for binding affinity measurement of the purified antibody to the purified $M$. tuberculosis LAM. Our data reveals that anti-LAM IgG has low $\mathrm{KD}$ values (below $10^{-8} \mathrm{M}$ ) which indicate that this antibody possess high affinity towards mycobacterial LAM (Figure S-3 and Table S-1). The affinity values support the high specificity and affinity of this antibody to LAM molecules.

Label-free immunoassay for LAM detection. A standard physical adsorption functionalisation in which the antibody is immobilised via hydrophobic and electrostatic interactions was selected for our new diagnostic platform. ${ }^{24}$ Physical adsorption 
involves two steps: the immobilisation of the antibody by electrostatic interactions and the blocking of the free sites to prevent non-specific adsorptions.

To pursue the biosensing evaluation, an optimisation with buffer is advised before using complex matrices, such as urine. The evaluation in buffer will allow us to study the performance of the biosensor platform for the specific TB biomarker without the interference of other proteins or compounds also present in urine.

Different immobilisation parameters must be optimised for the LAM detection in buffer. In order to provide a good antiLAM density with enough accessibility to LAM and to guarantee a suitable surface coverage for minimising non-specific adsorptions, various concentrations of the antibody and BSA, as blocking agent, were tested to improve the immobilisation performance. Each antibody concentration, ranging from 10 to 100 $\mu \mathrm{g} / \mathrm{mL}$, was evaluated at fixed conditions for pump speed (10 $\mu \mathrm{L} / \mathrm{min}$ ) and time (30 min). The surface coverage was analysed from the immobilisation signal. Immobilisation signals showed an increasing tendency with increasing antibody concentrations (data not shown). As antibody concentrations above $50 \mu \mathrm{g} / \mathrm{mL}$

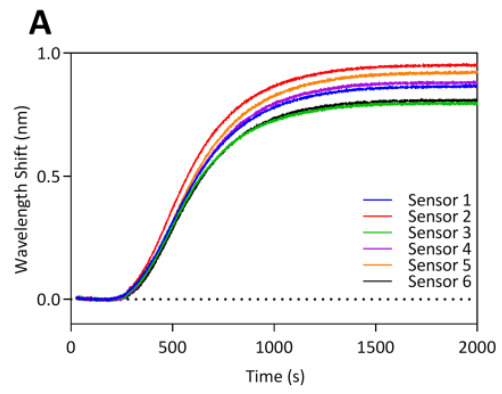

\section{B}

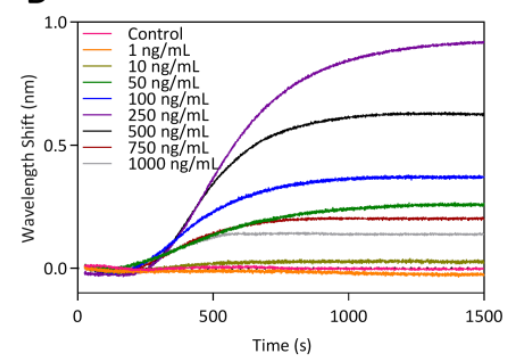

showed a similar signal, this means that the sensor surface was almost covered with $50 \mu \mathrm{g} / \mathrm{mL}$ of the antibody. After the antibody immobilisation, the same procedure was followed for the blocking step using different concentrations of BSA (from 1 to $10 \mathrm{mg} / \mathrm{mL}$ ) and analysing the detection signal of a non-specific protein (data not shown). A PBS solution with $10 \mathrm{mg} / \mathrm{mL}$ of BSA was the one that blocks the remaining free sensor surface areas and avoids non-specific adsorptions.

Using the optimised protocol, an antibody solution (50 $\mu \mathrm{g} / \mathrm{mL})$ in PBS was injected followed by a BSA solution (10 $\mathrm{mg} / \mathrm{mL}$ ). Milli-Q water was employed as running buffer for the immobilisation step The immobilisation step shows high reproducibility between different sensor cartridges (Coefficient of Variation $(\mathrm{CV}): 5 \%$ ). The running buffer was changed to PBS once the immobilisation process was finished and the target detection was then carried out. Target LAM samples were dissolved in PBS at concentrations ranging from 1 to $1000 \mathrm{ng} / \mathrm{mL}$ and injected into the system. All experiments with LAM in buffer were done by triplicate.

An example of real-time LAM detection $(250 \mathrm{ng} / \mathrm{mL})$

Figure 4. (A) Real-time sensogram showing the detection of $250 \mathrm{ng} / \mathrm{mL}$ of LAM in buffer. (B) Sensograms showing the LAM detection at different concentrations (from 1 to $1000 \mathrm{ng} / \mathrm{mL}$ of BSA protein) in buffer. (C) Calibration curve obtained for different LAM concentrations in buffer. Solid line corresponds to the one-site specific binding fit. All data show mean \pm SD of triplicate measurements. with the POC instrument is shown in Figure 4A. A simultaneous good response for all of the sensors contained in the same photonic sensor chip was observed.

As antibodies are not covalently attached to the sensor surface, it was not possible to regenerate the biosurface. For this reason, we have employed an additive immunoassay.

Successive dilutions of increasing LAM concentrations were injected in order to estimate the sensitivity, as shown in Figure 4B. For all of the concentrations evaluated, a clear sensor response was obtained. As expected with an additive assay, the antibody reaches a saturation level and loses its ability to detect more LAM, explaining the reduced sensor signal. For example, Figure 4B shows a higher LAM signal response for $250 \mathrm{ng} / \mathrm{mL}$ than for $1000 \mathrm{ng} / \mathrm{mL}$.

The selectivity of the biosensor was tested under the explained conditions for LAM detection, monitoring the wavelength shift after the injection of $1000 \mathrm{ng} / \mathrm{mL}$ of BSA protein, a non-specific protein for the immobilised antibody. As observed in Figure 4B (control), BSA protein leads to a negligible response of the biosensor.

A calibration curve of triplicates for LAM detection in buffer was plotted in Figure 4C, resulting in a LOD of $956 \mathrm{pg} / \mathrm{mL}$ equivalent to $54.63 \mathrm{pM}(\mathrm{R} 2=0.98)$, revealing the excellent sensitivity of the sensors and the selectivity of the antibodies against LAM, and overall indicating the good performance of the photonic chip and the prototype instrument.
For the evaluation of the reproducibility between sensors, the standard deviation was calculated for nine different detection signals by triplicate, obtaining a mean value of $40 \mathrm{pm}$. This result demonstrates that the variability between sensors is negligible.

LAM analysis in spiked urine samples. The main goal for a sensor applied to clinical diagnosis is the ability to detect a biomarker or a panel of biomarkers associated with the disease directly in human biological fluids, preferably obtained using a non-invasive technique. For this reason, the next step in our study was the evaluation of LAM directly in human urine. Urine is an ideal candidate for a POC platform due to its advantages in obtainment and storage.

We directly analysed LAM in real human urine samples, which is especially complex due to the undesired non-specific adsorption of different urine components. Several blocking agents can be employed to avoid or greatly decrease non-specific adsorption on the sensor surface. Previous studies have demonstrated that coating of the sensor surface with PLL-gPEG and the presence of surfactant agent Tween 20 in the detection buffer, minimises the background signal produced by undiluted urine. ${ }^{25-27}$ Based on this, we have studied the effect of different concentrations of blocking agents in order to reduce non-specific adsorption when flowing urine from a healthy patient over the sensors were we have previously immobilised the anti-LAM antibody: (i) $10 \mathrm{mg} / \mathrm{mL}$ of BSA, (ii) $0.5 \mathrm{mg} / \mathrm{mL}$ of 
PLL-g-PEG and (iii) $0.75 \mathrm{mg} / \mathrm{mL}$ of PLL-g-PEG and $10 \mathrm{mg} / \mathrm{mL}$ of BSA. First, an undiluted urine sample from a healthy donor was injected on the surface blocked with $10 \mathrm{mg} / \mathrm{mL}$ of BSA and resulted in a background signal of $1.27 \mathrm{~nm}$. Second, using 0.5 $\mathrm{mg} / \mathrm{mL}$ of PLL-g-PEG for the surface blocking, we observed a strong reduction of non-specific binding of undiluted urine with respect to BSA solution, but we cannot consider it as a negligible signal. In order to avoid this small signal, a combination of $0.75 \mathrm{mg} / \mathrm{mL}$ of PLL-g-PEG and $10 \mathrm{mg} / \mathrm{mL}$ of BSA was used to minimise non-specific adsorption between the urine components and the sensor surface to $80 \mathrm{pm}$. Thus, we will adopt this strategy for future evaluations of real urine samples and use PBS with $0.5 \%$ of Tween 20 as the running buffer for the evaluation of urine samples. When monitoring undiluted urine in real-time, a peak can be observed when the sample starts to flow in the sensing area (see Figure 5B), which we attribute to the use of PBST $0.5 \%$ as running buffer.

Diluted urine and undiluted urine were evaluated in order to observe possible interferences of the urine matrix in the additive assay. Spiked urine samples with different LAM concentrations were diluted 1:1 in PBS or injected directly without a pre-treatment onto the sensor. Figure 5 illustrates the different LAM concentrations in diluted and undiluted urine. Injection of urine from a healthy donor was performed, resulting in no background signal (see Figure 5) and confirming the absence of nonspecific adsorptions of urine components.

A

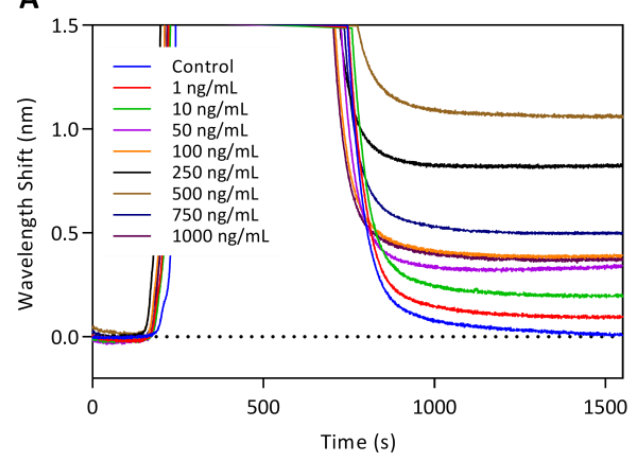

B

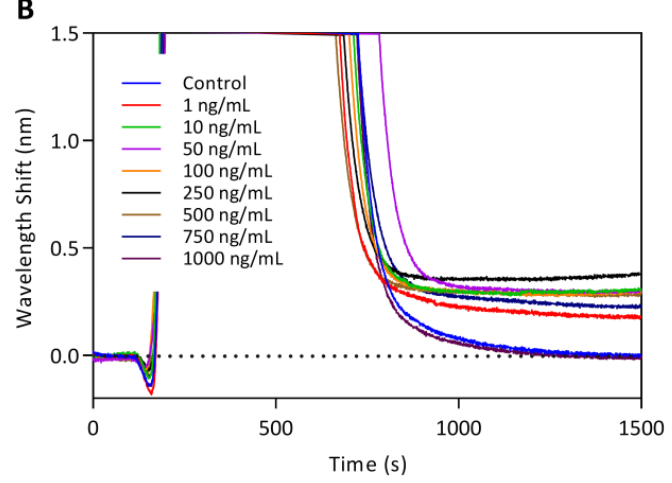

Figure 5. Sensograms showing the LAM detection at different concentrations (from 1 to $1000 \mathrm{ng} / \mathrm{mL}$ ) and control in (A) diluted and (B) undiluted urine.

A calibration curve of triplicates for LAM-spiked undiluted and diluted urine was plotted in Figure 6, resulting in a LOD of $731 \mathrm{pg} / \mathrm{mL}(41.77 \mathrm{pM}$; R2 = 0.97) and $475 \mathrm{pg} / \mathrm{mL}(27.14 \mathrm{pM}$; $\mathrm{R} 2=0.92)$, respectively. With reproducible and repetitive results, the curve of LAM-spiked undiluted urine showed higher sensitivity than in buffer and diluted urine (see Figure 6), suggesting that urine components create a more favourable environment for the antibody-antigen interaction.

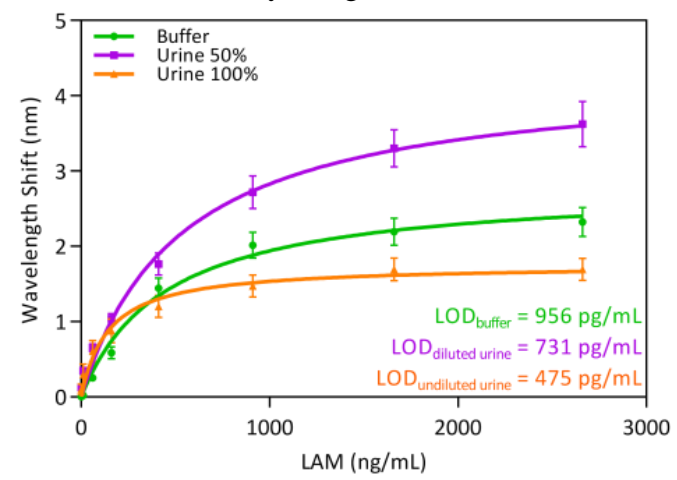

Figure 6. Calibration curves obtained for different LAM concentrations in buffer, diluted urine and undiluted urine. Solid line corresponds to the one-site specific binding fit of every curve. All data show mean \pm standard deviation of triplicate measurements.

The LOD in undiluted urine obtained with our new POC platform is better than those achieved using commercial lateral flow tests (LOD $10^{5}-10^{6} \mathrm{pM}$ ) and conventional ELISAs (LOD $\left.1.83 \cdot 10^{5} \mathrm{pM}\right)$, using different antibodies and immunoassay formats. ${ }^{28-30}$

The results confirmed the high sensitivity and specificity of our methodology, as well as the robustness of not only the antibodies but also the photonic sensor chips and the prototype instrument. In case that a positive signal is observed with this methodology, it would presumably represent the specific recognition of LAM by the antibodies immobilised on the sensor surface.

Validation with real urine samples. With the promising previous results in spiked urine, we were able to validate the prototype using a direct assay without the need of any pre-treatment of the human urine samples to be analysed. There are some parameters, such as $\mathrm{pH}$ and the concentration of different urine components, which can strongly vary between patients and which can have an effect in the evaluation.

With the optimal conditions previously selected for the evaluation of undiluted urine, we applied the POC platform to study the presence of LAM in the urine of 10 patients with confirmed TB from Tanzania and 10 healthy patients from Tanzania and Spain. The Tanzania samples were collected from hospitals and directly observed treatment short course (DOTS) centers, ${ }^{31}$ while the Spain samples were donated by volunteers.

Non-treated and undiluted real urine samples were directly flowed over the sensor chip using the in-flow system. Signals were monitored in real-time and converted into positive or negative TB diagnosis depending on the obtained LAM signal, as shown in Figure 7A. Based on the shifts from the optimisation process, patients were determined to be TB positive if the LAM signal was higher than $90 \mathrm{pm}$. Through evaluation of twenty real urine samples and taking into account this threshold, only one sample was identified as a false positive (see Figure 8). In our case, it is not important to determine the LAM concentration in urine, due to the fact that LAM is a cell wall component of $M$. tuberculosis and can only be present in the urine of those infected with the pathogenic bacteria. 
Positive results with the in-flow system allow the evaluation of the same real samples using the final configuration of the POC prototype which does not use the external pumps but employs a three-syringe system. With this configuration, two of the syringes contained PBST $0.5 \%$ and the other one the urine sample $(150 \mu \mathrm{L})$. An example of a real-time signal for the same samples as for the in-flow system is shown in Figure 7B. The resulted signal for healthy urine returns back to the baseline (see Figure 7B).

A comparison between both systems for the evaluation of two specific samples is shown in Figure 7. With both systems we cannot observe any significance difference in the absolute value of the obtained wavelength shift variation. This can also be observed in Figure 8 for the twenty urine samples analysed. The main difference between both systems was observed for Patient 4 (see Figure 8), who was the only false positive observed in our study when using the in-flow system. Taking account all these results, we can corroborate the high reproducibility and diagnosis capacity of our methodology.

A

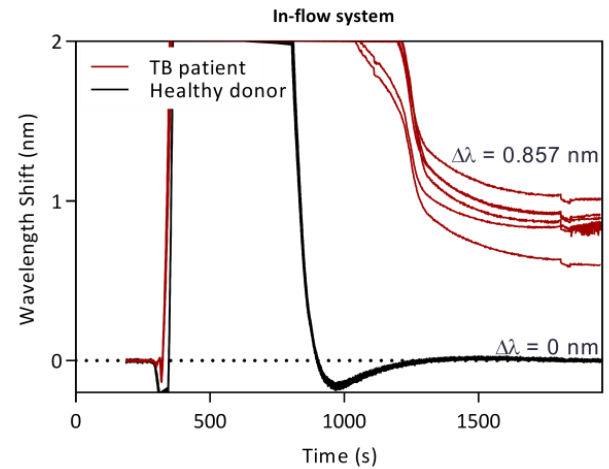

B

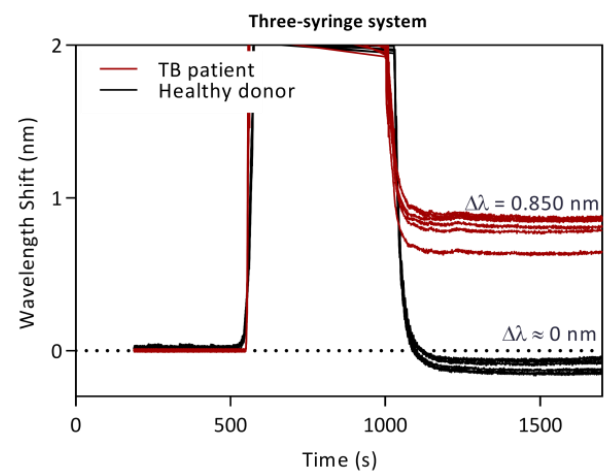

Figure 7. Real-time sensograms for the comparison of two real urine samples using (a) three-syringe and (B) in-flow systems.

One of the disadvantages of the current LAM detection methods in urine is that they are not able to detect TB in people without HIV. However, with our novel POC platform, the time to result is only 15 minutes for the classification of patients as healthy or infected, regardless of HIV coinfection, as shown in

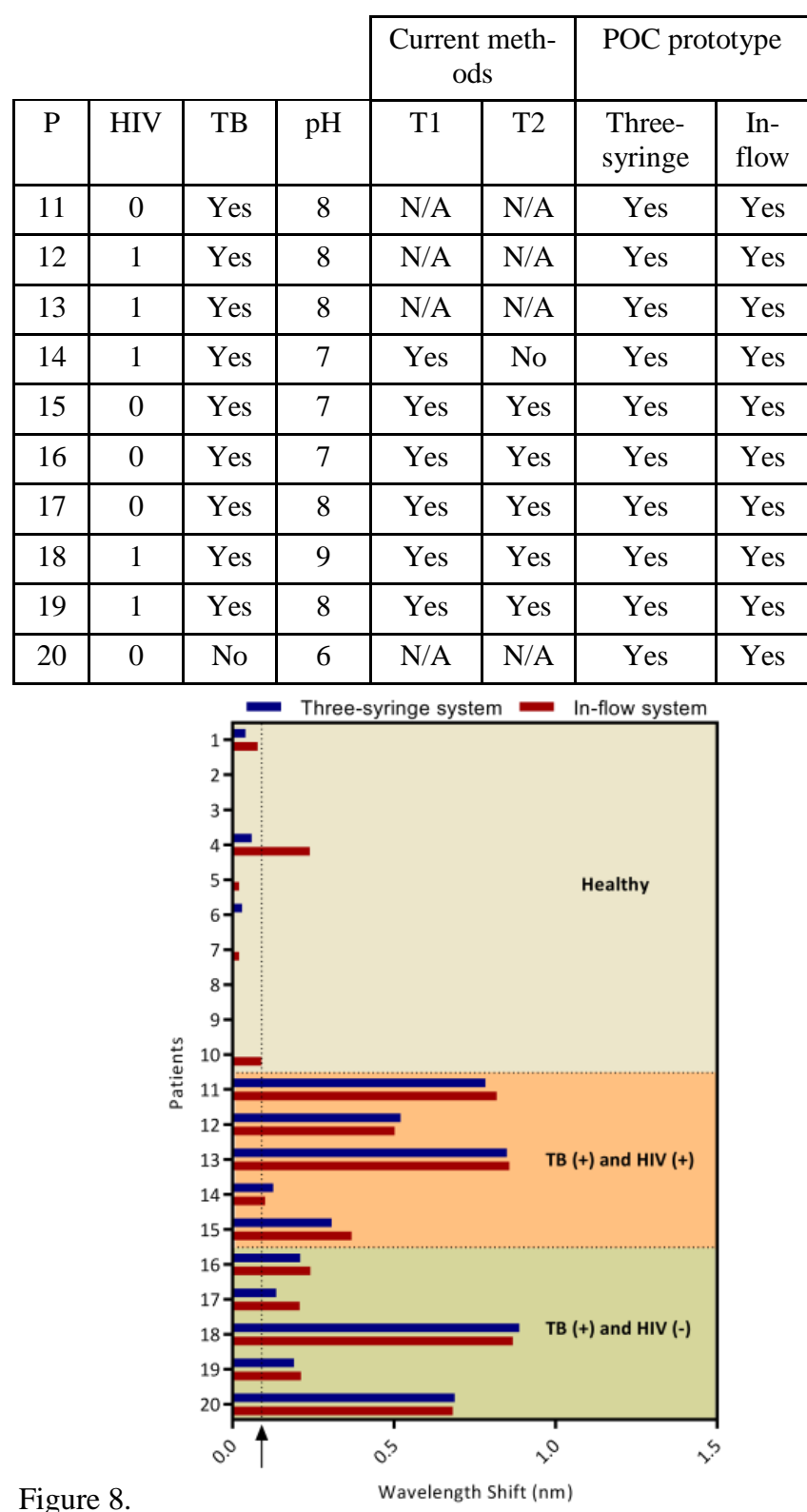

Figure 8.

Figure 8. Bar plot of the wavelength shift signals obtained from the analysis of twenty urine samples from healthy and TB patients with and without HIV using two different systems for the fluid injection. 
Black arrow shows the threshold to classify patients as healthy or infected.

Table 1 and 2 list and compare the results of the analysis obtained with the novel POC and with current techniques such as GeneXpert and sputum smear microscopy. For each patient, the values of $\mathrm{pH}$, HIV status and current TB diagnosis are reported. Results obtained with our platform show a good correlation to those obtained with the current methods as observed when compared the data in both tables. As illustrated in Figure 8 and Tables 1 and 2, out of the 20 real urine samples analysed by duplicate, 10 samples were true positive, 9 samples were true negative and when using the in-flow approach, only one sample was a false positive. Therefore, the validation of the novel POC platform using the final configuration with three-syringe showed a sensitivity of $100 \%$, a specificity of $100 \%$ and an area under the curve (AUC) of 1 as calculated by a ROC curve analysis (Figure S-4). This analysis was also carried out for the in-flow system and both systems together (Figure S-5 and S-6).

Table 1. Evaluation of urine samples from healthy volunteers with the POC prototype.

\begin{tabular}{|c|c|c|c|}
\cline { 3 - 4 } \multicolumn{2}{c|}{} & \multicolumn{2}{c|}{ POC prototype } \\
\hline $\begin{array}{c}\text { Healthy } \\
\text { donors }\end{array}$ & $\mathrm{pH}$ & $\begin{array}{c}\text { Three-sy- } \\
\text { ringe }\end{array}$ & $\begin{array}{c}\text { In- } \\
\text { flow }\end{array}$ \\
\hline 1 & 8 & No & No \\
\hline 2 & 8 & No & No \\
\hline 3 & 8 & No & No \\
\hline 4 & 7 & No & Yes \\
\hline 5 & 7 & No & No \\
\hline 6 & 7 & No & No \\
\hline 7 & 8 & No & No \\
\hline 8 & 9 & No & No \\
\hline 9 & 8 & No & No \\
\hline 10 & 6 & No & No \\
\hline
\end{tabular}

Table 2. Evaluation of urine samples from patients with confirmed TB using current methodologies (GeneXpert and Microscopy) in comparison with the POC prototype.

P: patients; HIV: HIV status; TB: TB diagnosis; T1: GeneXpert; T2: Microscopy;

\section{CONCLUSIONS}

We have developed a novel POC platform for the detection of a cell wall component of $M$. tuberculosis in undiluted urine based on a highly sensitive MZI sensor chip integrated in a polymer microfluidic cartridge. The strategy consists of a direct immunoassay for the label-free LAM detection in unprocessed urine in less than 15 minutes. Several parameters have been optimised, reaching a limit of detection as low as $475 \mathrm{pg} / \mathrm{mL}$ $(27.14 \mathrm{pM})$ for the direct detection in spiked urine samples and corroborating the excellent selectivity and sensitivity of the produced antibodies. The validation of the methodology with clinical samples from TB patients and healthy donor samples allows the detection of TB in people with and without HIV coinfection and the results show excellent correlation to those obtained with GeneXpert, and sputum microscopy techniques. The platform has shown a high sensitivity (100\%) and specificity $(100 \%)$ in comparison with rapid tests commercially available for TB detection. The diagnosis of people only infected with
TB has not been possible with the current rapid tests on the market, providing more advantages to our POC instrument. The advantages added to our instrument (low cost, user friendliness, results in 15 minutes, no requirement for qualified personnel for operation and no need of special laboratory infrastructure) make this POC platform an outstanding candidate for in-situ use in primary doctor's office and patient's bedsides. We therefore demonstrated the high sensitivity, specificity and applicability of our platform, which could be used during routine check-ups in developing countries.

As the instrument is designed for the simultaneous detection of a panel of six different biomarkers, this open the door for the use of our POC platform for the fast detection of different diseases, such as diabetes, cancer, HIV, stroke, heart diseases, dementias diseases, malaria, hepatitis A, respiratory infections, and kidney diseases, among others.

\section{ASSOCIATED CONTENT}

Supporting Information. Additional text and figures showing the reagents, ethics statement, data analysis and complete prototype instrument, as well as further description of the monoclonal antibody and target production, studying the affinity and providing kinetics properties of the monoclonal antibody. Also, there is the Receiver Operating Characteristic (ROC) analysis for the diagnostic performance of our platform.

\section{AUTHOR INFORMATION}

\section{Corresponding Author \\ *E-mail: laura.lechuga@icn2.cat. Tel: +34 937374620}

\section{Author Contributions}

The manuscript was written through contributions of all authors. All authors have given approval to the final version of the manuscript.

\section{ACKNOWLEDGMENT}

This work was funded by the European Commission under the FP7 project Pocket (grant 610389). The ICN2 is funded by the CERCA programme/Generalitat de Catalunya. The ICN2 is supported by the Severo Ochoa programme of the Spanish Ministry of Economy, Industry and Competitiveness (MINECO, Grant no. SEV-20130295).

\section{REFERENCES}

(1) WHO. Global Tuberculosis Report 2017; 2017.

(2) Srivastava, S. K.; van Rijn, C. J. M.; Jongsma, M. A. BiosensorBased Detection of Tuberculosis. RSC Adv. 2016, 6 (22), 1775917771.

(3) Pai, M.; Behr, M. A.; Dowdy, D.; Dheda, K.; Divangahi, M.; Boehme, C. C.; Ginsberg, A.; Swaminathan, S.; Spigelman, M.; Getahun, H.; et al. Tuberculosis. Nat. Rev. Dis. Prim. 2016, 2.

(4) Shah, M.; Chihota, V.; Coetzee, G.; Churchyard, G.; Dorman, S. E. Comparison of Laboratory Costs of Rapid Molecular Tests and Conventional Diagnostics for Detection of Tuberculosis and DrugResistant Tuberculosis in South Africa. BMC Infect. Dis. 2013, 13 (1).

(5) Callaway, E. Improved Diagnostics Fail to Halt the Rise of Tuberculosis. Nature 2017, 551 (7681), 424-425.

(6) Viñuelas-Bayón, J.; Vitoria, M. A.; Samper, S. Rapid Diagnosis of Tuberculosis. Detection of Drug Resistance Mechanisms. Enfermedades Infecc. y Microbiol. Clin. (English ed.) 2017, 35 (8), 518-526. 
(7) Goletti, D.; Petruccioli, E.; Joosten, S. A.; Ottenhoff, T. H. M. Tuberculosis Biomarkers: From Diagnosis to Protection. Infect. Dis. Rep. 2016, 8 (2), 24-32.

(8) Dheda, K.; Barry, C. E.; Maartens, G. Tuberculosis. Lancet 2016, 387 (10024), 1211-1226.

(9) Bekmurzayeva, A.; Sypabekova, M.; Kanayeva, D. Tuberculosis Diagnosis Using Immunodominant, Secreted Antigens of Mycobacterium Tuberculosis. Tuberculosis 2013, 93 (4), 381-388.

(10) Woldeyohannes, D.; Sisay, S.; Mengistu, B.; Kassa, H. Directly Observed Treatment Short-Course (DOTS) for Treatment of New Tuberculosis Cases in Somali Regional State, Eastern Ethiopia: Ten Years Retrospective Study. BMC Res. Notes 2015, 8 (1), 1-7.

(11) Shete, P. B.; Ravindran, R.; Chang, E.; Worodria, W.; Chaisson, L. H.; Andama, A.; Davis, J. L.; Luciw, P. A.; Huang, L.; Khan, I. H.; et al. Evaluation of Antibody Responses to Panels of M. Tuberculosis Antigens as a Screening Tool for Active Tuberculosis in Uganda. PLoS One 2017, 12 (8), 1-12.

(12) Peter, J.; Green, C.; Hoelscher, M.; Mwaba, P.; Zumla, A.; Dheda, K. Urine for the Diagnosis of Tuberculosis: Current Approaches, Clinical Applicability, and New Developments. Curr. Opin. Pulm. Med. 2010, 16 (3), 262-270.

(13) Hamasur, B.; Bruchfeld, J.; Haile, M.; Pawlowski, A.; Bjorvatn, B.; Källenius, G.; Svenson, S. B. Rapid Diagnosis of Tuberculosis by Detection of Mycobacterial Lipoarabinomannan in Urine. J. Microbiol. Methods 2001, 45 (1), 41-52.

(14) World Health Organization. The Use of Lateral Flow Urine Lipoarabinomannan Assay (LF-LAM) for the Diagnosis and Screening of Active Tuberculosis in People Living with HIV: Policy. 2015, 1-74.

(15) Agha, M. A.; El-Helbawy, R. H.; El-Helbawy, N. G.; El-Sheak, N. M. Utility of Quantitative Analysis of Urine Lipoarabinomannan in the Diagnosis of Tuberculosis. Egypt. J. Chest Dis. Tuberc. 2013, 62 (3), 401-407.

(16) Minion, J.; Leung, E.; Talbot, E.; Dheda, K.; Pai, M.; Menzies, D. Diagnosing Tuberculosis with Urine Lipoarabinomannan: Systematic Review and Meta-Analysis. Eur. Respir. J. 2011, 38 (6), $1398-1405$

(17) Mukundan, H.; Price, D. N.; Goertz, M.; Parthasarathi, R.; Montaño, G. A.; Kumar, S.; Scholfield, M. R.; Anderson, A. S.; Gnanakaran, S.; Iyer, S.; et al. Understanding the Interaction of Lipoarabinomannan with Membrane Mimetic Architectures. Tuberculosis 2012, 92 (1), 38-47.

(18) Mishra, A. K.; Driessen, N. N.; Appelmelk, B. J.; Besra, G. S. Lipoarabinomannan and Related Glycoconjugates: Structure, Biogenesis and Role in Mycobacterium Tuberculosis Physiology and Host-Pathogen Interaction. FEMS Microbiol. Rev. 2011, 35 (6), 11261157.

(19) Fukuda, T.; Matsumura, T.; Ato, M.; Hamasaki, M.; Nishiuchi, Y.; Murakami, Y.; Maeda, Y.; Yoshimori, T.; Matsumoto, S.; Kobayashi, K.; et al. Critical Roles for Lipomannan and
Lipoarabinomannan in Cell Wall Integrity of Mycobacteria and Pathogenesis of Tuberculosis. MBio 2013, 4 (1), 8-10.

(20) Paris, L.; Magni, R.; Zaidi, F.; Araujo, R.; Saini, N.; Harpole, M.; Coronel, J.; Kirwan, D. E.; Steinberg, H.; Gilman, R. H.; et al. Urine Lipoarabinomannan Glycan in HIV-Negative Patients with Pulmonary Tuberculosis Correlates with Disease Severity. 2017, 1-12.

(21) Lawn, S. D. Point-of-Care Detection of Lipoarabinomannan (LAM) in Urine for Diagnosis of HIV-Associated Tuberculosis: A State of the Art Review. BMC Infect. Dis. 2012, 12 (1), 1.

(22) D. Martens, P. Ramirez-Priego, M. S. Murib, A.A. Elamin, A.B. Gonzalez-Guerrero, M. Stehr, F. Jonas, B. Anton, N. Hlawatsch, P. Soetaert, R. Vos, A. Stassen, S. Severi, W. Van Roy, R. Bockstaele, H. Becker, M. Singh, L.M. Lechuga, P. B. Low-Cost Integrated Biosensing Platform Based on SiN Nanophotonics for Biomarker Detection in Urine. Anal. Methods 2018, In-press.

(23) González-Guerrero, A. B.; Maldonado, J.; Herranz, S. Lechuga, L. M. Trends in Photonic Lab-on-Chip Interferometric Biosensors for Point-of-Care Diagnostics. Anal. Methods 2016, 8 (48), 8380-8394.

(24) Welch, N. G.; Scoble, J. A.; Muir, B. W.; Pigram, P. J. Orientation and Characterization of Immobilized Antibodies for Improved Immunoassays (Review). Biointerphases 2017, 12 (2), $02 \mathrm{D} 301$.

(25) Soler, M.; Estevez, M.-C.; Villar-Vazquez, R.; Casal, J. I.; Lechuga, L. M. Label-Free Nanoplasmonic Sensing of TumorAssociate Autoantibodies for Early Diagnosis of Colorectal Cancer. Anal. Chim. Acta 2016, 930, 31-38.

(26) Gutiérrez-Mejía, F. A.; van Ijzendoorn, L. J.; Prins, M. W. J. Surfactants Modify the Torsion Properties of Proteins: A Single Molecule Study. N. Biotechnol. 2015, 32 (5), 441-449.

(27) Soler, M.; Estevez, M.-C.; Alvarez, M.; Otte, M. A.; Sepulveda, B.; Lechuga, L. M. Direct Detection of Protein Biomarkers in Human Fluids Using Site-Specific Antibody Immobilization Strategies. Sensors (Basel). 2014, 14 (2), 2239-2258.

(28) Mukundan, H.; Kumar, S.; Price, D. N.; Ray, S. M.; Lee, Y. J.; Min, S.; Eum, S.; Kubicek-Sutherland, J.; Resnick, J. M.; Grace, W. K.; et al. Rapid Detection of Mycobacterium Tuberculosis Biomarkers in a Sandwich Immunoassay Format Using a Waveguide-Based Optical Biosensor. Tuberculosis 2012, 92 (5), 407-416.

(29) Shah, M.; Variava, E.; Holmes, C. B.; Coppin, A.; Golub, J. E.; McCallum, J.; Wong, M.; Luke, B.; Martin, D. J.; Chaisson, R. E.; et al. Diagnostic Accuracy of a Urine Lipoarabinomannan Test for Tuberculosis in Hospitalized Patients in a High HIV Prevalence Setting. JAIDS J. Acquir. Immune Defic. Syndr. 2009, 52 (2), 145-151.

(30) Shah, M.; Martinson, N. A.; Chaisson, R. E.; Martin, D. J.; Variava, E.; Dorman, S. E. Quantitative Analysis of a Urine-Based Assay for Detection of Lipoarabinomannan in Patients with Tuberculosis. J. Clin. Microbiol. 2010, 48 (8), 2972-2974.

(31) WHO. World Health Organization, What Is DOTS (Directly Observed Treatment, Short Course). SEARO 2017.

Table of Contents (TOC) 


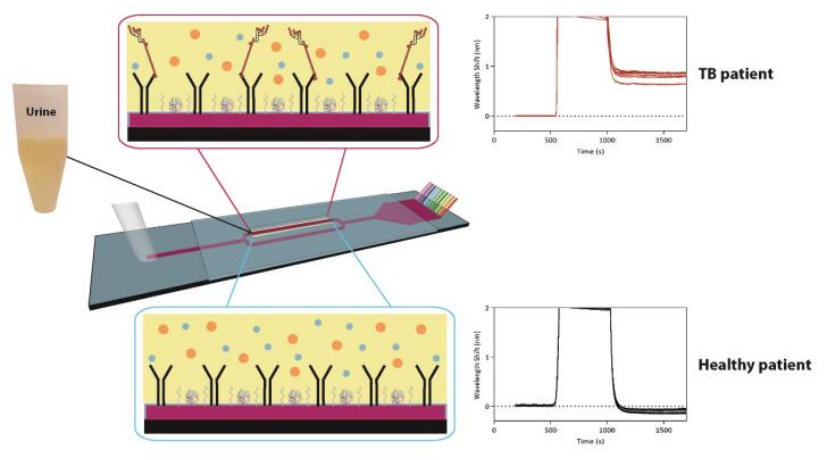

